\title{
Domino Metathesis Involving ROM-RCM of Substituted Norbornenes. Rapid Access to Densely Functionalized Tricyclic Bridged and Condensed Ring systems
}

Chanchal K. Malik and Subrata Ghosh*

Department of Organic Chemistry, Indian Association for the Cultivation of Science, Jadavpur, Kolkata 700 032, India

\section{Supporting Information}




\section{Table of contents}

1. Experimental Procedure and spectral data ------------------------------------- S3

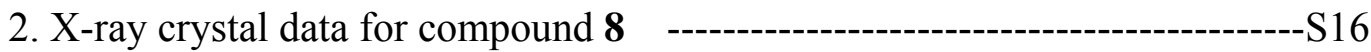

3. ${ }^{1} \mathrm{H},{ }^{13} \mathrm{C}$ NMR and DEPT of compound 8----------------------------------------S18- S19

4. ${ }^{1} \mathrm{H},{ }^{13} \mathrm{C}$ NMR and DEPT of compound 15 --------------------------------------S 19-S20

7. ${ }^{1} \mathrm{H},{ }^{13} \mathrm{C}$ NMR and DEPT of compound 21 -------------------------------------S21- S22

8. 9. ${ }^{1} \mathrm{H},{ }^{13} \mathrm{C}$ NMR and DEPT of compound 28 -------------------------------------S22- S23

10. ${ }^{1} \mathrm{H},{ }^{13} \mathrm{C}$ NMR and DEPT of compound 33 \& 34 ------------------------------S24- S25

11. $1 \mathrm{H},{ }^{13} \mathrm{C}$ NMR and DEPT of compound 35------------------------------------ S25- S26

12. $1 \mathrm{H},{ }^{13} \mathrm{C}$ NMR and DEPT of Compound 36------------------------------------S27-S28 


\section{Experimental Section:}

All reactions were carried out under a blanket of Ar. Melting points were taken in open capillaries in sulfuric acid bath and are uncorrected. Petroleum ether refers to the fraction having bp $60-80^{\circ} \mathrm{C}$. A usual work up of the reaction mixture consists of extraction with ether, washing with brine, drying over anhydrous $\mathrm{Na}_{2} \mathrm{SO}_{4}$, and removal of the solvent in vacuo. Column chromatography was carried out with silica gel (60-120 mesh). Peak position in ${ }^{1} \mathrm{H}$ and ${ }^{13} \mathrm{C}$ NMR spectra are indicated in ppm downfield from internal TMS in $\delta$ units. NMR spectra were taken in $\mathrm{CDCl}_{3}$ at $300 \mathrm{MHz}$ for ${ }^{1} \mathrm{H}$ and $75 \mathrm{MHz}$ for ${ }^{13} \mathrm{C} .{ }^{13} \mathrm{C}$ peaks assignment is based on DEPT experiment. IR spectra were recorded as neat for liquid and in $\mathrm{KBr}$ for solids.

Methyl 3-(bicyclo[2.2.1]hept-5-en-2-yl)-3-oxopropanoate 2. A $100 \mathrm{~mL}$ three necked round bottomed flask is equipped with a mechanical stirrer, reflux condenser and a pressure equalizing dropping funnel bearing a nitrogen inlet. The flask is flushed with nitrogen and charged with dimethyl carbonate $(9.3 \mathrm{~mL}, 110.29 \mathrm{mmol})$, THF $(25 \mathrm{~mL})$ and sodium hydride $(3.3 \mathrm{~g}, 138.1 \mathrm{mmol})$. The suspension is stirred and heated at reflux temperature and the ketone 1 (6 g, $44.12 \mathrm{mmol})$ in THF $(15 \mathrm{~mL})$ was added drop wise over a period of $30 \mathrm{~min}$. The mixture was stirred and heated at reflux for another $1 \mathrm{~h}$, cooled in an ice bath for $10 \mathrm{~min}$ and hydrolyzed by slowly adding $12 \mathrm{~mL} 3(\mathrm{M})$ aqueous acetic acid. The contents of the flask were poured into $20 \mathrm{~mL}$ aqueous sodium chloride and the aqueous mixture was extracted with $3 \times 100 \mathrm{~mL}$ portion of $\mathrm{CHCl}_{3}$. The organic layer was dried and the solvent was removed under reduced pressure. The compound was purified by column chromatography ether- petroleum ether (1:6) as eluent to afford the $\beta$ - keto ester $2(5.6 \mathrm{~g}, 60 \%)$ as a colorless viscous liquid: IR (neat) $v_{\max } 1708 \mathrm{~cm}^{-1}, 1747$ $\mathrm{cm}^{-1} ;{ }^{1} \mathrm{H}$ NMR $\delta 1.32(1 \mathrm{H}, \mathrm{d}, J=8.0 \mathrm{~Hz}), 1.38-1.52(2 \mathrm{H}, \mathrm{m}), 1.73-1.81(1 \mathrm{H}, \mathrm{m}), 2.91$ $(1 \mathrm{H}$, br s), $3.15(1 \mathrm{H}, \mathrm{m}), 3.23(1 \mathrm{H}$, br s), $3.46(2 \mathrm{H}, \mathrm{d}, J=3.42 \mathrm{~Hz}), 3.71(3 \mathrm{H}, \mathrm{s}), 5.85$ $(1 \mathrm{H}, \mathrm{dd}, J=5.4,2.6 \mathrm{~Hz}), 6.16(1 \mathrm{H}, \mathrm{dd}, J=5.4,2.9 \mathrm{~Hz}) ;{ }^{13} \mathrm{C} \mathrm{NMR} \delta 27.6\left(\mathrm{CH}_{2}\right), 42.7$ $(\mathrm{CH}), 45.9(\mathrm{CH}), 48.3\left(\mathrm{CH}_{2}\right), 49.9\left(\mathrm{CH}_{2}\right), 52.0(\mathrm{CH}), 52.2\left(\mathrm{OCH}_{3}\right), 131.2(\mathrm{CH}), 138.2$ (CH), 167.7 (COOMe), $202.8(\mathrm{CO})$; HRMS (ESI) m/z $(\mathrm{M}+\mathrm{Na})^{+}$calcd for $\mathrm{C}_{11} \mathrm{H}_{14} \mathrm{O}_{3} \mathrm{Na}$ : 217.0841, found: 217.0850 .

Synthesis of the compound 3. To a magnetically stirred suspension of $\mathrm{NaH}(0.300 \mathrm{~g}$, $12.9 \mathrm{mmol}, 60 \%$ in oil, freed from adhering oil by repeated washing with petroleum 
ether) in dry THF $(6 \mathrm{~mL})$ at $0^{\circ} \mathrm{C}$ was added dropwise a solution of the compound $2(1.25$ $\mathrm{g}, 6.44 \mathrm{mmol})$ in THF $(10 \mathrm{~mL})$ under $\mathrm{N}_{2}$ atmosphere. To this mixture was added HMPA $(0.5 \mathrm{~mL})$ followed by allyl bromide $(1.4 \mathrm{~mL}, 13.9 \mathrm{mmol})$ drop wise. After stirring for an additional $12 \mathrm{~h}$ at $\mathrm{rt}$, the reaction mixture was cooled at $0^{\circ} \mathrm{C}$ and quenched by adding cold water $(2 \mathrm{~mL})$. Usual work up of the reaction mixture followed by column chromatography using ether-petroleum ether (1:9) as eluent afforded the diallyl derivative 3 (1.46 g, 83\%); IR (neat) $v_{\max } 1708.0,1747 \mathrm{~cm}^{-1} ;{ }^{1} \mathrm{H}$ NMR $\delta 1.19-1.24(2 \mathrm{H}, \mathrm{m}), 1.36$ $(1 \mathrm{H}, \mathrm{d}, J=8.2 \mathrm{~Hz}), 1.65-1.79(1 \mathrm{H}, \mathrm{m}), 2.63(4 \mathrm{H}, \mathrm{d}, J=10.4 \mathrm{~Hz}), 2.86(1 \mathrm{H}$, br s), 2.94 $(1 \mathrm{H}, \mathrm{br} \mathrm{s}), 3.14-3.19(1 \mathrm{H}, \mathrm{m}), 3.71(3 \mathrm{H}, \mathrm{s}), 5.02-5.09(4 \mathrm{H}, \mathrm{m}), 5.41-5.60(2 \mathrm{H}, \mathrm{m}), 5.78$ $(1 \mathrm{H}, \mathrm{dd}, J=5.5,2.8 \mathrm{~Hz}), 6.19(1 \mathrm{H}, \mathrm{dd}, J=5.5,3.1 \mathrm{~Hz}),{ }^{13} \mathrm{C} \mathrm{NMR} \delta 31.9\left(\mathrm{CH}_{2}\right), 35.65$ $\left(\mathrm{CH}_{2}\right), 35.7\left(\mathrm{CH}_{2}\right), 42.9(\mathrm{CH}), 47.1(\mathrm{CH}) 47.9(\mathrm{CH}), 50.2\left(\mathrm{CH}_{2}\right), 52.1(\mathrm{OMe}), 63.3(\mathrm{C})$, $119.1\left(\mathrm{CH}_{2}\right), 131.3(\mathrm{CH}), 132.1(\mathrm{CH}), 132.4(\mathrm{CH}), 137.4(\mathrm{CH}), 172.1$ ( COOMe), 207.4 (CO); HRMS (ESI) m/z (M+ Na) ${ }^{+}$calcd for $\mathrm{C}_{17} \mathrm{H}_{22} \mathrm{O}_{3} \mathrm{Na}: 297.1467$, found: 297.1459.

Synthesis of the compound 4. A solution of the ketone 3 (1.5 g, $5.47 \mathrm{mmol})$ in THF (6 $\mathrm{mL}$ ) was added drop wise to a magnetically stirred solution of LDA [prepared from diisopropyl amine $(1.9 \mathrm{~mL}, 13.67 \mathrm{mmol})$ in anhydrous THF $(5 \mathrm{~mL})$ and $\mathrm{n}-\mathrm{BuLi}(5.7 \mathrm{~mL}$, $13.67 \mathrm{mmol}, 2.4 \mathrm{M}$ in hexane] at $-78{ }^{\circ} \mathrm{C}$. The reaction mixture was then slowly raised to $30{ }^{\circ} \mathrm{C}$ and stirred at that temperature for $1 \mathrm{~h}$. The reaction mixture was then again cooled to $-78{ }^{\circ} \mathrm{C}$ and to it HMPA $(1.5 \mathrm{~mL})$ was added followed by addition of allyl bromide (1.5 $\mathrm{mL}, 13.67 \mathrm{mmol})$. The reaction mixture was allowed to attain $\mathrm{rt}$ and stirred for $2 \mathrm{~h}$. After quenching with saturated aqueous $\mathrm{NH}_{4} \mathrm{Cl}$ solution $(1 \mathrm{~mL})$, the reaction mixture was worked up in the usual way to afford after column chromatography [ether-petroleum ether 1:9] the ketone $4(1.12 \mathrm{~g}, 65 \%)$; IR: $v_{\max } 1685,1732 \mathrm{~cm}^{-1} ;{ }^{1} \mathrm{H}$ NMR $\delta 1.31(1 \mathrm{H}, \mathrm{dd}$, $J=9,1.8 \mathrm{~Hz}) ; 1.39(1 \mathrm{H}, \mathrm{dd}, J=12.3,3.6 \mathrm{~Hz}) ; 1.51(1 \mathrm{H}, \mathrm{d}, J=9 \mathrm{~Hz}) ; 1.78(1 \mathrm{H}, \mathrm{dd}, J=$ 12.3, $2.4 \mathrm{~Hz}) ; 2.26(1 \mathrm{H}, \mathrm{dd}, J=15,7.8 \mathrm{~Hz}) ; 2.39(1 \mathrm{H}, \mathrm{dd}, J=9,3 \mathrm{~Hz}) ; 2.44-2.56(2 \mathrm{H}$, m); $2.62(1 \mathrm{H}, \mathrm{dd}, J=14.1,7.2 \mathrm{~Hz}) ; 2.79(1 \mathrm{H}, \mathrm{br} \mathrm{s}) ; 2.93(1 \mathrm{H}, \mathrm{dd}, J=15,6.6 \mathrm{~Hz}) ; 3.11$ $(1 \mathrm{H}, \mathrm{br} \mathrm{s}) ; 3.69(3 \mathrm{H}, \mathrm{s}) ; 4.94-5.01(6 \mathrm{H}, \mathrm{d}, J=16.9 \mathrm{~Hz}) ; 5.46-5.59(2 \mathrm{H}, \mathrm{m}) ; 5.68-5.73$ $(1 \mathrm{H}, \mathrm{m}) ; 5.96(1 \mathrm{H}, \mathrm{dd}, J=5.6,2.9 \mathrm{~Hz}) ; 6.07(1 \mathrm{H}, \mathrm{dd}, J=5.4,3 \mathrm{~Hz}) ; \mathrm{HRMS}(\mathrm{ESI}) \mathrm{m} / \mathrm{z}$ $(\mathrm{M}+\mathrm{H})^{+}$calcd for $\mathrm{C}_{20} \mathrm{H}_{27} \mathrm{O}_{3}: 315.1960$, found: 315.1955 .

Metathesis of the compound 4. Synthesis of the bridged tricyclic ketone 8. A magnetically stirred solution of the compound $4(70 \mathrm{mg}, 0.223 \mathrm{mmol})$ in dry 
dichloromethane $(15 \mathrm{ml})$ at $-78{ }^{\circ} \mathrm{C}$ was saturated by purging ethylene through it. After 30 min Grubbs' catalyst 7 (18 mg, $0.022 \mathrm{mmol}, 10 \mathrm{~mol} \%$ ) was added to it. After $2 \mathrm{~h}$ the reaction was found to be complete (TLC) and the solvent was removed under vacuum. The residual mass was charged on to a column of silica gel (60 - 120 mesh) containing a bed of neutral alumina on top of silica gel and chromatographed (15\% ether - hexane) to afford the compound 8 (38 mg, 59\%); m.p. 79-80 ${ }^{\circ} \mathrm{C}$; IR: $v_{\max }=1737.7,1687.6 \mathrm{~cm}^{-1} ;{ }^{1} \mathrm{H}$ NMR $\delta 1.48(1 \mathrm{H}, \mathrm{ddd}, J=13.4,8.3,4.7 \mathrm{~Hz}), 1.71(1 \mathrm{H}, \mathrm{dd}, J=13.4,7.1 \mathrm{~Hz}), 1.91(1 \mathrm{H}$, dd, $J=13.2,11.4 \mathrm{~Hz}), 2.09(1 \mathrm{H}, \mathrm{d}, J=14.1 \mathrm{~Hz}), 2.27-2.41(\mathrm{~m}, 2 \mathrm{H}), 2.45-2.56(\mathrm{~m}, 2 \mathrm{H})$, 2.59-2.70 (m, 2H), $2.96(1 \mathrm{H}, \mathrm{ddd}, J=14.4,6.3,1.2 \mathrm{~Hz}), 3.20-3.23(1 \mathrm{H} \mathrm{m}), 3.73(3 \mathrm{H}, \mathrm{s}$, COOMe), $4.89(1 \mathrm{H}, \mathrm{dt}, J=10.8,0.75 \mathrm{~Hz}), 4.99(1 \mathrm{H}, \mathrm{dt}, J=17.1,1.4 \mathrm{~Hz}), 5.46(1 \mathrm{H}, \mathrm{ddd}$, $J=9.9,4.5,1.8 \mathrm{~Hz}) 5.71-5.86(2 \mathrm{H}, \mathrm{m}), 5.89-5.93(2 \mathrm{H}, \mathrm{m}),{ }^{13} \mathrm{C} \mathrm{NMR} \delta=31.7\left(\mathrm{CH}_{2}\right)$, $32.6\left(\mathrm{CH}_{2}\right), 35.2\left(\mathrm{CH}_{2}\right), 38.8\left(\mathrm{CH}_{2}\right), 39.6(\mathrm{CH}), 41.9\left(\mathrm{CH}_{2}\right), 42.4(\mathrm{CH}), 52.3\left(\mathrm{OCH}_{3}\right)$, 64.77(C), 64.81(C), $113.2\left(\mathrm{CH}_{2}\right), 128.9(\mathrm{CH}), 129.6(\mathrm{CH}), 129.8(\mathrm{CH}), 138.2(\mathrm{CH})$, $142.7(\mathrm{CH}), 174.0(\mathrm{COOMe}), 211.0(\mathrm{CO})$; HRMS (ESI) $\mathrm{m} / \mathrm{z}(\mathrm{M}+\mathrm{H})^{+}$calcd. for $\mathrm{C}_{18} \mathrm{H}_{22} \mathrm{O}_{3}: 287.1647$, found $287.1641 \mathrm{~m} / \mathrm{z}$ and a fraction $(20 \mathrm{mg}$ ) having an inseparable mixture of several components as revealed by the presence of several COOMe signals in its ${ }^{1} \mathrm{H}$ NMR spectrum.

Methyl 2-allylbicyclo[2.2.1]hept-5-ene-2-carboxylate 11. Following the procedure used for allylation of the ester 3, the ester 10 (4 g, $26.32 \mathrm{mmol})$ was treated with LDA [prepared from diisopropylamine $(9.2 \mathrm{~mL}, 65.79 \mathrm{mmol})$ in THF $(10 \mathrm{~mL})$ and $\mathrm{n}-\mathrm{BuLi}$ (28.6 $\mathrm{mL}, 65.79 \mathrm{mmol}, 2.3 \mathrm{M}$ in hexane], HMPA $(2 \mathrm{~mL})$ and allyl bromide $(8.4 \mathrm{~mL}$, $78.96 \mathrm{mmol})$ to afford the ester $\mathbf{1 1}(3.97 \mathrm{~g}, 78 \%)$ as a colorless liquid; IR: (neat): $v_{\max }$ $1737 \mathrm{~cm}^{-1} ;{ }^{1} \mathrm{H}$ NMR $\delta$ 1.31-1.56 (3H, m), $1.86(1 \mathrm{H}, \mathrm{dd}, J=12,2.7 \mathrm{~Hz}), 2.33(1 \mathrm{H}, \mathrm{dd}, J=$ 13.7, $7.5 \mathrm{~Hz}), 2.84(2 \mathrm{H}, \mathrm{br} \mathrm{s}), 3.57(3 \mathrm{H}, \mathrm{s}), 4.97-5.03(2 \mathrm{H}, \mathrm{m}), 5.64-5.75(1 \mathrm{H}, \mathrm{m}), 5.96$ $(1 \mathrm{H}, \mathrm{dd}, J=5.7,3 \mathrm{~Hz}), 6.14(1 \mathrm{H}, \mathrm{dd}, J=5.6,2.9 \mathrm{~Hz}) ;{ }^{13} \mathrm{C} \mathrm{NMR} \delta 35.6\left(\mathrm{CH}_{2}\right) ; 42.6(\mathrm{CH})$; $44.5\left(\mathrm{CH}_{2}\right) ; 47.0\left(\mathrm{CH}_{2}\right) ; 5.01(\mathrm{CH}) ; 51.3\left(\mathrm{OCH}_{3}\right) ; 54.7(\mathrm{C}) ; 116.9\left(\mathrm{CH}_{2}\right) ; 134.5(\mathrm{CH})$; $134.6(\mathrm{CH}) ; 138.4(\mathrm{CH}) ; 176.2(\mathrm{COOMe})$; HRMS (ESI) $\mathrm{m} / \mathrm{z}(\mathrm{M}+\mathrm{Na})^{+}$calcd. for $\mathrm{C}_{12} \mathrm{H}_{16} \mathrm{NaO}_{2:}$ : 215.1048, found $215.1055 \mathrm{~m} / \mathrm{z}$.

2-Allylbicyclo[2.2.1] hept-5-ene-2-carbaldehyde 12. To a stirred suspension of $\mathrm{LiAIH}_{4}$ $(1.78 \mathrm{~g}, 46.88 \mathrm{mmol})$ in diethyl ether $(15 \mathrm{~mL})$ at $0^{\circ} \mathrm{C}$ was added drop wise a solution of the norbornene ester $\mathbf{1 1}(4.5 \mathrm{~g}, 23.4 \mathrm{mmol})$ in diethyl ether $(15 \mathrm{~mL})$. After stirring at that 
temperature for $1 \mathrm{~h}$ the reaction mixture was quenched by sequential addition of water $(1.8 \mathrm{~mL})$, aqueous $\mathrm{NaOH}$ solution $(15 \%, 1.8 \mathrm{~mL})$ and water $(5.4 \mathrm{~mL})$ and allowed to attain rt. After stirring for $15 \mathrm{~min}$, the white solid formed was filtered off after washing it thoroughly with diethyl ether. Removal of solvent in vacuo followed by column chromatography using ether-petroleum ether (1:5) as eluent afforded the corresponding alcohol (3.4 g, 90\%) as a colorless viscous liquid; IR: $v_{\max } 3367 \mathrm{~cm}^{-1}$; ${ }^{1} \mathrm{H}$ NMR $\delta 1.36-$ $1.48(2 \mathrm{H}, \mathrm{m}), 1.59(2 \mathrm{H}, \mathrm{d}, J=8.7 \mathrm{~Hz}), 2.29(1 \mathrm{H}, \mathrm{dd}, J=13.8,7.1 \mathrm{~Hz}), 2.46(1 \mathrm{H}, \mathrm{dd}, J=$ $13.9,7.7 \mathrm{~Hz}), 2.62-2.63(1 \mathrm{H}, \mathrm{m}), 2.78-2.79(1 \mathrm{H}, \mathrm{m}), 3.25(1 \mathrm{H}, \mathrm{dd}, J=10.8,4.2 \mathrm{~Hz})$, $3.37(1 \mathrm{H}, \mathrm{dd}, J=10.8,3.4 \mathrm{~Hz}), 5.03-5.14(2 \mathrm{H}, \mathrm{m}), 5.86-5.97(1 \mathrm{H}, \mathrm{m}), 6.0-6.1(2 \mathrm{H}, \mathrm{m})$; ${ }^{13} \mathrm{C}$ NMR $\delta 34.8\left(\mathrm{CH}_{2}\right), 41.7\left(\mathrm{CH}_{2}\right), 42.5(\mathrm{CH}), 47.3\left(\mathrm{CH}_{2}\right), 47.29(\mathrm{C}), 48.2(\mathrm{CH}), 67.5$ $\left(\mathrm{CH}_{2}\right), 116.7\left(\mathrm{CH}_{2}\right), 135.1(\mathrm{CH}), 136.4(\mathrm{CH}), 136.8(\mathrm{CH}), \mathrm{HRMS}(\mathrm{ESI}) \mathrm{m} / \mathrm{z}(\mathrm{M}+\mathrm{Na})^{+}$ calcd. for $\mathrm{C}_{11} \mathrm{H}_{16} \mathrm{NaO}$ : 187.1099 , found $187.1095 \mathrm{~m} / \mathrm{z}$. The hydroxy compound thus obtained was oxidized as follows.

To a magnetically stirred cold $\left(-78^{\circ} \mathrm{C}\right)$ solution of oxalyl chloride $(2.7 \mathrm{~mL}, 31.09$ mmol) in dichloromethane $(10 \mathrm{~mL})$ was added a solution of DMSO $(3.67 \mathrm{~mL}, 31.09$ $\mathrm{mmol})$ in dichloromethane $(5 \mathrm{~mL})$. A solution of the above alcohol $(3.4 \mathrm{~g}, 20.73 \mathrm{mmol})$ in dichloromethane $(1 \mathrm{~mL})$ was added to this mixture and stirred for $45 \mathrm{~min}$. Triethylamine $(11.6 \mathrm{~mL}, 82.9 \mathrm{mmol})$ was then added to it drop wise. The reaction mixture was allowed to attain rt. It was then cooled to $0^{\circ} \mathrm{C}$ and quenched by adding water. Usual work-up of the reaction mixture followed by column chromatography using ether: petroleum ether (1:19) as eluent afforded the aldehyde $12(2.35 \mathrm{~g}, 70 \%)$ as a colorless liquid; IR: $v_{\max } 1720 \mathrm{~cm}^{-1}$; ${ }^{1} \mathrm{H}$ NMR $\delta$ 1.47-1.76 (3H, m), 2.34-2.43 (2H, m), 2.59 - 2.61 $(1 \mathrm{H}, \mathrm{m}), 2.86(1 \mathrm{H}, \mathrm{br} \mathrm{s}), 2.92(1 \mathrm{H}, \mathrm{br} \mathrm{s}), 4.97-5.07(2 \mathrm{H}, \mathrm{m}), 5.63-5.77(1 \mathrm{H}, \mathrm{m}), 6.04$ $(1 \mathrm{H}, \mathrm{dd}, J=5.2,2.8 \mathrm{~Hz}), 6.18(1 \mathrm{H}, \mathrm{dd}, J=5.5,2.8 \mathrm{~Hz}), 9.42(1 \mathrm{H}, \mathrm{s}) ;{ }^{13} \mathrm{C}$ NMR $\delta 33.9$ $\left(\mathrm{CH}_{2}\right), 40.8\left(\mathrm{CH}_{2}\right), 43.2(\mathrm{CH}), 47.3\left(\mathrm{CH}_{2}\right), 49.9(\mathrm{CH}), 58.6(\mathrm{C}), 117.6\left(\mathrm{CH}_{2}\right), 133.5(\mathrm{CH})$, $134.1(\mathrm{CH}), 138.7(\mathrm{CH}), 205.7(\mathrm{CHO})$, HRMS (ESI) $\mathrm{m} / \mathrm{z}(\mathrm{M}+\mathrm{Na})^{+}$calcd. for $\mathrm{C}_{11} \mathrm{H}_{14} \mathrm{NaO}: 185.0942$, found 185.0939 .

1-(2-Allylbicyclo[2.2.1]hept-5-en-2-yl)pent-4-en-1-one 13. To a magnetically stirred solution of 4-butenyl magnesium bromide [prepared from 4-bromo-1- butene $(1.75 \mathrm{~mL}$, $17.28 \mathrm{mmol})$ in THF $(10 \mathrm{~mL})$ and magnesium turnings (476 $\mathrm{mg}, 19.9 \mathrm{mmol})$ ] was added a solution of the aldehyde $12(1.4 \mathrm{~g}, 8.6 \mathrm{mmol})$ in THF $\left(10 \mathrm{~mL}\right.$ at $0^{\circ} \mathrm{C}$. Stirring was 
continued for $1 \mathrm{~h}$. On cooling to $0{ }^{\circ} \mathrm{C}$ the reaction mixture was quenched by adding water $(1 \mathrm{~mL})$ and filtered. Organic phase was washed with brine $(3 \times 6 \mathrm{~mL})$ and dried. Removal of solvent in vacuo followed by column chromatography using ether-petroleum ether (1:5) as eluent afforded the addition product $(1.26 \mathrm{~g}, 67 \%)$ as a mixture of two diastereomers; IR: $v_{\max } 3378 \mathrm{~cm}^{-1}$; ${ }^{1} \mathrm{H}$ NMR (for the mixture) $\delta 1.26-1.33(2 \mathrm{H}, \mathrm{m}), 1.34-$ $1.43(2 \mathrm{H}, \mathrm{m}), 1.52-1.65(4 \mathrm{H}, \mathrm{m}), 2.26-2.35(2 \mathrm{H}, \mathrm{m}), 2.48-2.56(2 \mathrm{H}, \mathrm{m}), 2.78(1 \mathrm{H}, \mathrm{br} \mathrm{s})$, $2.93(1 \mathrm{H}, \mathrm{br} \mathrm{s}), 3.17(1 \mathrm{H}, \mathrm{t}, J=6.2 \mathrm{~Hz}), 4.93-5.14(4 \mathrm{H}, \mathrm{m}), 5.82(1 \mathrm{H}, \mathrm{m}), 6.1-6.19(3 \mathrm{H}$, m); ${ }^{13} \mathrm{C}$ NMR $\delta 31.5\left(\mathrm{CH}_{2}\right), 31.9\left(\mathrm{CH}_{2}\right), 32.9\left(\mathrm{CH}_{2}\right), 33.1\left(\mathrm{CH}_{2}\right), 35.7\left(\mathrm{CH}_{2}\right), 37.1\left(\mathrm{CH}_{2}\right)$, 40.7, $\left(\mathrm{CH}_{2}\right) 40.8\left(\mathrm{CH}_{2}\right), 42.4(\mathrm{CH}), 42.9(\mathrm{CH}), 46.8\left(\mathrm{CH}_{2}\right), 47.9\left(\mathrm{CH}_{2}\right), 47.6(\mathrm{CH}), 50.3$ $(\mathrm{CH}), 50.2(\mathrm{C}), 50.9(\mathrm{C}), 78.7(\mathrm{CHOH}), 79.6(\mathrm{CH}-\mathrm{OH}), 114.6\left(\mathrm{CH}_{2}\right), 114.8\left(\mathrm{CH}_{2}\right), 116.3$ $\left(\mathrm{CH}_{2}\right), 116.5\left(\mathrm{CH}_{2}\right), 134.3(\mathrm{CH}), 135.2(\mathrm{CH}), 137.1(\mathrm{CH}), 137.4(\mathrm{CH}), 137.8(\mathrm{CH}), 137.8$ $(\mathrm{CH}) ; 138.5(\mathrm{CH}), 138.8(\mathrm{CH})$; HRMS (ESI) $\mathrm{m} / \mathrm{z}(\mathrm{M}+\mathrm{Na})^{+}$calcd for $\mathrm{C}_{15} \mathrm{H}_{22} \mathrm{ONa}$ :241.1568, found:241.1560. To a magnetically stirred and cooled $\left(0{ }^{\circ} \mathrm{C}\right)$ solution of the mixture of the addition products obtained as above $(400 \mathrm{mg}, 2.31 \mathrm{mmol})$ in acetone (2 $\mathrm{mL}$ ) was added drop wise Jones reagent ${ }^{1}(1 \mathrm{~mL})$ till the color of the reagent persisted. After stirring at that temperature for an additional $30 \mathrm{~min}$, the reaction mixture was diluted with water $(2 \mathrm{~mL})$, extracted with diethyl ether $(2 \times 10 \mathrm{~mL})$. Combined organic layer was washed subsequently with $2 \%$ aqueous $\mathrm{NaOH}$ solution $(5 \mathrm{~mL})$, brine $(5 \mathrm{~mL})$ and dried. Removal of the solvent in vacuo followed by column chromatography using ether-petroleum ether (1: 9) as eluent afforded the ketone 13 (421 mg, 85\%); IR: $v_{\max }$ $1705 \mathrm{~cm}^{-1}$; ${ }^{1} \mathrm{H}$ NMR $\delta$ 1.44-1.49 (2H, m), $1.63(1 \mathrm{H}, \mathrm{d}, J=8.7 \mathrm{~Hz}), 1.96(1 \mathrm{H}, \mathrm{dd}, J=12.1$, $2.6 \mathrm{~Hz}), 2.24-2.51(4 \mathrm{H}, \mathrm{m}), 2.59-2.62(2 \mathrm{H}, \mathrm{m}), 2.85(1 \mathrm{H}, \mathrm{br} \mathrm{s}), 2.88(1 \mathrm{H}, \mathrm{br} \mathrm{s}), 4.94-$ $5.06(4 \mathrm{H}, \mathrm{m}), 5.61(1 \mathrm{H}, \mathrm{m}), 5.80(1 \mathrm{H}, \mathrm{m}), 5.93(1 \mathrm{H}, \mathrm{dd}, J=5.7,2.8 \mathrm{~Hz}) ; 6.13(1 \mathrm{H}, \mathrm{dd}, J$ $=5.6,3.0 \mathrm{~Hz}) ;{ }^{13} \mathrm{C} \mathrm{NMR} \delta 27.8\left(\mathrm{CH}_{2}\right), 34.0\left(\mathrm{CH}_{2}\right), 38.9\left(\mathrm{CH}_{2}\right), 42.8(\mathrm{CH}), 43.9\left(\mathrm{CH}_{2}\right)$, $47.3\left(\mathrm{CH}_{2}\right), 50.3(\mathrm{CH}), 60.7(\mathrm{C}), 114.9\left(\mathrm{CH}_{2}\right), 117.1\left(\mathrm{CH}_{2}\right), 133.8(\mathrm{CH}), 134.2(\mathrm{CH})$, $137.7(\mathrm{CH}), 138.6(\mathrm{CH}), 211.6(\mathrm{CO})$; HRMS (ESI) $\mathrm{m} / \mathrm{z}(\mathrm{M}+\mathrm{Na})^{+}$calcd for $\mathrm{C}_{15} \mathrm{H}_{20} \mathrm{ONa}$ :239.1412, found: 239.1417 .

2-Allyl-1-(2-allylbicyclo[2.2.1]hept-5-en-2-yl)pent-4-en-1-one 14. A solution of the ketone 13 (262 $\mathrm{mg}, 1.2 \mathrm{mmol})$ in THF ( $3 \mathrm{~mL})$ was added drop wise to a magnetically stirred solution of LDA [prepared from diiosopropylamine $(0.25 \mathrm{~mL}, 1.8 \mathrm{mmol})$ in

\footnotetext{
${ }^{1}$ Bowden, K.; Halsall, T. G.; Jones, E. R. H.; Weedon, B. C. L. J. Chem. Soc. 1946, 39 - 45.
} 
anhydrous THF $(3 \mathrm{~mL})$ and $\mathrm{n}-\mathrm{BuLi}(1.2 \mathrm{~mL}, 1.8 \mathrm{mmol}, 1.5 \mathrm{M}$ in hexane $]$ at $-78^{\circ} \mathrm{C}$. The reaction mixture was then slowly raised to $-30^{\circ} \mathrm{C}$ and stirred at that temperature for $1 \mathrm{~h}$. The reaction mixture was then again cooled to $-78^{\circ} \mathrm{C}$ and to it HMPA $(0.2 \mathrm{~mL})$ was added followed by addition of allyl bromide $(0.2 \mathrm{~mL}, 1.82 \mathrm{mmol})$. The reaction mixture was allowed to attain $\mathrm{rt}$ and stirred for $2 \mathrm{~h}$. After quenching with saturated aqueous $\mathrm{NH}_{4} \mathrm{Cl}$ solution $(1 \mathrm{~mL})$, the reaction mixture was worked up in the usual way to afford after column chromatography [ether-petroleum ether 1:19) the ketone 14 (227 $\mathrm{mg}, 73 \%$ ); IR: $v_{\max } 1697 \mathrm{~cm}^{-1} ;{ }^{1} \mathrm{H}$ NMR $\delta$ 1.56-1.60 $(2 \mathrm{H}, \mathrm{m}), 1.80(1 \mathrm{H}, \mathrm{dd}, J=12.0,1.7 \mathrm{~Hz}), 1.96$ $2.07(2 \mathrm{H}, \mathrm{m}), 2.09-2.31(3 \mathrm{H}, \mathrm{m}), 2.50(2 \mathrm{H}, \mathrm{d}, J=7.05 \mathrm{~Hz}), 2.69(1 \mathrm{H}, \mathrm{m}), 2.84(1 \mathrm{H}, \mathrm{br}$ s), $2.94\left(1 \mathrm{H}\right.$, br s), $4.97-5.05(6 \mathrm{H}, \mathrm{m}), 5.59-5.74(3 \mathrm{H}, \mathrm{m}), 6.02-6.09(2 \mathrm{H}, \mathrm{m}) ;{ }^{13} \mathrm{C}$ NMR $\delta 35.4\left(\mathrm{CH}_{2}\right), 35.5\left(\mathrm{CH}_{2}\right), 35.6\left(\mathrm{CH}_{2}\right), 42.7(\mathrm{CH}), 43.6\left(\mathrm{CH}_{2}\right), 46.8\left(\mathrm{CH}_{2}\right), 47.8$ $(\mathrm{CH}), 49.0(\mathrm{CH}), 61.8(\mathrm{C}) 117.3\left(\mathrm{CH}_{2}\right), 117.3\left(\mathrm{CH}_{2}\right), 117.7\left(\mathrm{CH}_{2}\right), 134.6(\mathrm{CH}), 135.9$ (CH), $136.3(\mathrm{CH}), 137.3(\mathrm{CH}), 137.5(\mathrm{CH}), 215.5(\mathrm{CO})$; HRMS (ESI) m/z (M + Na) calcd for $\mathrm{C}_{18} \mathrm{H}_{24} \mathrm{ONa}: 279.1725$, found: 179.1732 .

Metathesis of the compound 14. Synthesis of the bridged tricyclic ketone 15. Following the procedure described for metathesis of the compound 5, the compound 14 ( $100 \mathrm{mg}, 0.39 \mathrm{mmol})$ in DCM $(15 \mathrm{~mL})$ with the catalyst $7(20 \mathrm{mg}, 0.024 \mathrm{mmol})$ afforded the tricyclic compound 15 (54 mg, 60\%): IR: $v_{\max } 1699 \mathrm{~cm}^{-1} ;{ }^{1} \mathrm{H}$ NMR $\delta$ 146-1.54 (2H, m), $1.64(2 \mathrm{H}, \mathrm{d}, J=8.6 \mathrm{~Hz}), 1.93(2 \mathrm{H}, \mathrm{dd}, J=12.1,2.6 \mathrm{~Hz}), 2.41-2.55$ (3H, m), 2.67 (1H, dd, $J=14.6,7.1 \mathrm{~Hz}), 2.85(1 \mathrm{H}$, br s), $2.91(1 \mathrm{H}, \mathrm{br} \mathrm{s}), 3.48(1 \mathrm{H}, \mathrm{m}), 4.99-5.05(2 \mathrm{H}, \mathrm{m})$, 5.55-5.66 (3H, m), $5.96(1 \mathrm{H}, \mathrm{dd}, J=5.6,3.0 \mathrm{~Hz}), 6.12(1 \mathrm{H}, \mathrm{dd}, J=5.7,2.8 \mathrm{~Hz}) ;{ }^{13} \mathrm{C}$ NMR $\delta 33.8\left(\mathrm{CH}_{2}\right), 38.1\left(\mathrm{CH}_{2}\right), 38.8\left(\mathrm{CH}_{2}\right), 42.8(\mathrm{CH}), 43.2\left(\mathrm{CH}_{2}\right), 45.2(\mathrm{CH}), 47.2$ $\left(\mathrm{CH}_{2}\right), 49.9(\mathrm{CH}), 61.6(\mathrm{C}) ; 117.3\left(\mathrm{CH}_{2}\right), 128.3(\mathrm{CH}), 129.2(\mathrm{CH}), 134.6(\mathrm{CH}), 138.2$ (CH), 216.9 (CO); HRMS (ESI) m/z (M + Na) calcd for $\mathrm{C}_{16} \mathrm{H}_{20} \mathrm{ONa}: 251.1412$, found: 251.1406.

Methyl 2-(1-hydroxyallyl)bicyclo[2.2.1]hept-5-ene-2-carboxylate 16. A solution of the methyl ester $10(1.7 \mathrm{~g}, 11.18 \mathrm{mmol})$ in THF $(10 \mathrm{~mL})$ was added drop wise to a magnetically stirred solution of LDA [prepared from diisopropyl amine $(3.1 \mathrm{~mL}, 22.4$ $\mathrm{mmol})$ in anhydrous THF $(10 \mathrm{~mL})$ and $\mathrm{n}-\mathrm{BuLi}(15.9 \mathrm{~mL}, 22.4 \mathrm{mmol}), 1.4 \mathrm{M}$ in hexane] at $-78^{\circ} \mathrm{C}$. The reaction mixture was then slowly raised to $-30^{\circ} \mathrm{C}$ and stirred at that temperature for $1 \mathrm{~h}$. The reaction mixture was then again cooled to $-78^{\circ} \mathrm{C}$ and to it 
HMPA $(1 \mathrm{~mL})$ was added followed by acrolein $(2.3 \mathrm{~mL}, 33.5 \mathrm{mmol})$ drop wise. The reaction mixture was allowed to stir for $1 \mathrm{~h}$ at $-78^{\circ} \mathrm{C}$. After quenching with saturated aqueous $\mathrm{NH}_{4} \mathrm{Cl}$ solution $(1 \mathrm{~mL})$, the reaction mixture was worked up in the usual way to afford after column chromatography (ether-petroleum ether 1:4) one diastereoisomer $\left(\mathrm{R}_{\mathrm{f}}\right.$ 0.3 ) of the mixture the hydroxy-esters 16 (600mg, $42 \%$ ); IR: $v_{\max } 1720,3483 \mathrm{~cm}^{-1} ;{ }^{1} \mathrm{H}$ NMR $\delta$ 1.39-1.45 $(1 \mathrm{H}, \mathrm{m}), 1.67(1 \mathrm{H}, \mathrm{d}, J=8.8 \mathrm{~Hz}), 1.80(1 \mathrm{H}, \mathrm{dd}, J=12.3,2.7 \mathrm{~Hz}), 2.01$ $(1 \mathrm{H}, \mathrm{dd}, J=12.4,3.5 \mathrm{~Hz}), 2.47(1 \mathrm{H}, \mathrm{br} \mathrm{s} \mathrm{OH}), 2.88(1 \mathrm{H}, \mathrm{m}), 2.94(1 \mathrm{H}, \mathrm{m}), 3.61(3 \mathrm{H}, \mathrm{s})$, $4.17(1 \mathrm{H}, \mathrm{m}), 5.17-5.28(2 \mathrm{H}, \mathrm{m}), 5.91-6.03(1 \mathrm{H}, \mathrm{m}), 6.06(1 \mathrm{H}, \mathrm{dd}, J=5.6,2.9 \mathrm{~Hz})$, $6.21(1 \mathrm{H}, \mathrm{dd}, J=5.6,3.0 \mathrm{~Hz}) ;{ }^{13} \mathrm{CNMR} \delta 34.9\left(\mathrm{CH}_{2}\right), 42.2(\mathrm{CH}), 46.7\left(\mathrm{CH}_{2}\right), 47.7(\mathrm{CH})$, $51.4(\mathrm{CH}), 60.1(\mathrm{C}), 79.0(\mathrm{CH}), 116.3\left(\mathrm{CH}_{2}\right), 135.7(\mathrm{CH}), 137.6(\mathrm{CH}), 139.0(\mathrm{CH}), 175.0$ (COOMe); HRMS (ESI) $\mathrm{m} / \mathrm{z}(\mathrm{M}+\mathrm{Na})^{+}$calcd for $\mathrm{C}_{12} \mathrm{H}_{16} \mathrm{O}_{3} \mathrm{Na}$ :231.0997, found: 231.0987 and the other diastereoisomer $\left(\mathrm{R}_{\mathrm{f}} 0.35\right)(600 \mathrm{mg}, 42 \%)$; IR: $v_{\max } 1718,3478$ $\mathrm{cm}^{-1} ;{ }^{1} \mathrm{H}$ NMR $\delta 0.83(1 \mathrm{H}, \mathrm{dd}, J=12.3,2.5 \mathrm{~Hz}), 1.29(1 \mathrm{H}, \mathrm{d}, J=8.7 \mathrm{~Hz}), 1.42(1 \mathrm{H}, \mathrm{d}, \mathrm{J}$ $=8.7 \mathrm{~Hz}), 2.44(1 \mathrm{H}, \mathrm{dd}, J=12.3,3.9 \mathrm{~Hz}), 2.87(1 \mathrm{H}, \mathrm{br} \mathrm{s}), 3.19(1 \mathrm{H}, \mathrm{d}, J=10.4 \mathrm{~Hz}), 3.24$ $(1 \mathrm{H}, \mathrm{br} \mathrm{s}), 3.57(1 \mathrm{H}, \mathrm{br} \mathrm{s} \mathrm{OH}), 3.68(3 \mathrm{H}, \mathrm{s}), 5.05-5.25(2 \mathrm{H}, \mathrm{m}), 5.70-5.81(1 \mathrm{H}, \mathrm{m})$, $6.19-6.25(2 \mathrm{H}, \mathrm{m}) ;{ }^{13} \mathrm{CNMR} \delta 32.8\left(\mathrm{CH}_{2}\right), 42.1(\mathrm{CH}), 47.0\left(\mathrm{CH}_{2}\right), 49.8(\mathrm{CH}), 52(\mathrm{CH})$, $59.6(\mathrm{C}), 77.4\left(\mathrm{CH}_{3}\right), 115.1\left(\mathrm{CH}_{2}\right), 133.7(\mathrm{CH}), 137.4(\mathrm{CH}), 138.9(\mathrm{CH}), 179.4$ (COOMe); HRMS (ESI) $\mathrm{m} / \mathrm{z}(\mathrm{M}+\mathrm{Na})^{+}$calcd for $\mathrm{C}_{12} \mathrm{H}_{16} \mathrm{O}_{3} \mathrm{Na}$ :231.099, found: 231.0985 .

Methyl 2-(1-(methoxymethoxy)allyl)bicyclo[2.2.1]hept-5-ene-2-carboxylate 17. To a magnetically stirred solution of the alcohol $\mathbf{1 6}$ (with $\mathrm{R}_{\mathrm{f}} 0.3$ ) (500 mg, $2.4 \mathrm{mmol}$ ) in dimethoxymethane $(0.26 \mathrm{~mL}, 2.9 \mathrm{mmol})$ and dry DCM $(2 \mathrm{~mL}), \mathrm{BF}_{3} . \mathrm{OEt}_{2}(0.34 \mathrm{~mL}, 2.9$ mmol) was added drop wise at $0^{\circ} \mathrm{C}$. After complete addition, the reaction mixture was stirred for $30 \mathrm{~min}$ at $0^{\circ} \mathrm{C}$. After quenching with aqueous saturated $\mathrm{NaHCO}_{3}$ solution, the reaction mixture was worked up in the usual way to afford after column chromatography (ether-petroleum ether 1:9) The norbornene derivative 17 (0.91 g, 80\%); IR(neat): $v_{\max }$ $1741 \mathrm{~cm}^{-1}$; ${ }^{1} \mathrm{H}$ NMR $\delta 1.36(1 \mathrm{H}, \mathrm{dd}, J=8.6,1.4 \mathrm{~Hz}), 1.72(1 \mathrm{H}, \mathrm{d}, J=8.4 \mathrm{~Hz}), 1.78(1 \mathrm{H}$, $\mathrm{dd}, J=12.2,3.5 \mathrm{~Hz}), 2.01(1 \mathrm{H}, \mathrm{dd}, J=12.3,2.7 \mathrm{~Hz}), 2.86(1 \mathrm{H}, \mathrm{br} \mathrm{s}), 2.92(1 \mathrm{H}, \mathrm{br} \mathrm{s})$, $3.37(3 \mathrm{H}, \mathrm{s}), 3.6(3 \mathrm{H}, \mathrm{s}), 4.15(1 \mathrm{H}, \mathrm{d}, J=9 \mathrm{~Hz}), 4.48(1 \mathrm{H}, \mathrm{d}, J=6.9 \mathrm{~Hz}), 4.68(1 \mathrm{H}, \mathrm{d}, J=$ $6.5 \mathrm{~Hz}), 5.15-5.29(2 \mathrm{H}, \mathrm{m}), 5.76-5.88(1 \mathrm{H}, \mathrm{m}), 5.92(1 \mathrm{H}, \mathrm{dd}, J=5.4,3 \mathrm{~Hz}), 6.22(1 \mathrm{H}, \mathrm{dd}$, $J=5.4,3 \mathrm{~Hz}) ;{ }^{13} \mathrm{C}$ NMR $\delta 34.5\left(\mathrm{CH}_{2}\right), 42.3(\mathrm{CH}), 47.1\left(\mathrm{CH}_{2}\right), 49.2(\mathrm{CH}), 51.4\left(\mathrm{OCH}_{3}\right)$, 
$55.8\left(\mathrm{OCH}_{3}\right.$ of $\left.\mathrm{MOM}\right), 58.6(\mathrm{C}), 83.5(\mathrm{CH}), 93.3\left(\mathrm{OCH}_{2}\right), 119.5\left(\mathrm{CH}_{2}\right), 134.6(\mathrm{CH})$, $135.3(\mathrm{CH}), 139.9(\mathrm{CH}), 174.4(\mathrm{CO})$; HRMS (ESI) $\mathrm{m} / \mathrm{z}(\mathrm{M}+\mathrm{Na})^{+}$calcd for $\mathrm{C}_{14} \mathrm{H}_{20} \mathrm{O}_{4} \mathrm{Na}$ :275.1259, found: 275.1257 .

2-(1-(Methoxymethoxy)allyl)bicyclo[2.2.1]hept-5-ene-2-carbaldehyde 18. Following the procedure described for reduction of the ester 11, the ester 17 (400 $\mathrm{mg}, 1.695 \mathrm{mmol})$ in dry ether $(15 \mathrm{~mL})$ was reduced with $\mathrm{LiAlH}_{4}(128.8 \mathrm{mg}, 3.4 \mathrm{mmol})$ to afford the hydroxy compound $(0.807 \mathrm{~g}, 85 \%)$; IR: $v_{\max } 3417 \mathrm{~cm}^{-1} ;{ }^{1} \mathrm{H}$ NMR $\delta 1.22(1 \mathrm{H}, \mathrm{d}, J=12.3$ $\mathrm{Hz}), 1.39(1 \mathrm{H}, \mathrm{d}, J=8.1 \mathrm{~Hz}), 1.66(1 \mathrm{H}, \mathrm{d}, J=8.6 \mathrm{~Hz}), 1.72(1 \mathrm{H}, \mathrm{dd}, J=12.3,3.7 \mathrm{~Hz})$, $2.64(1 \mathrm{H}$, br s), $2.89(1 \mathrm{H}, \mathrm{br} \mathrm{s}), 3.03(1 \mathrm{H}$, br s $\mathrm{OH}), 3.41(3 \mathrm{H}, \mathrm{s}), 3.65(2 \mathrm{H}, \mathrm{d}, J=10.9$ $\mathrm{Hz}), 4.16(1 \mathrm{H}, \mathrm{d}, J=8.3 \mathrm{~Hz}), 4.53(1 \mathrm{H}, \mathrm{d}, J=6.8 \mathrm{~Hz}), 4.71(1 \mathrm{H}, \mathrm{d}, J=6.8 \mathrm{~Hz}), 5.22-$ $5.37(2 \mathrm{H}, \mathrm{m}), 5.92-6.01(1 \mathrm{H}, \mathrm{m}), 6.04(1 \mathrm{H}, \mathrm{dd}, J=5.8,2.9 \mathrm{~Hz}), 6.20(1 \mathrm{H}, \mathrm{dd}, J=5.6$, $2.9 \mathrm{~Hz}) ;{ }^{13} \mathrm{C}$ NMR $\delta 34.4\left(\mathrm{CH}_{2}\right), 43.2(\mathrm{CH}), 46.7(\mathrm{CH}), 47.0\left(\mathrm{CH}_{2}\right), 51.5(\mathrm{C}), 56.4\left(\mathrm{CH}_{3}\right)$, $67.9\left(\mathrm{CH}_{2}\right), 87.0(\mathrm{CH}), 94.3\left(\mathrm{CH}_{2}\right), 119.9\left(\mathrm{CH}_{2}\right), 135.4(\mathrm{CH}), 135.9(\mathrm{CH}), 138.5(\mathrm{CH})$; HRMS (ESI) $\mathrm{m} / \mathrm{z}(\mathrm{M}+\mathrm{Na})^{+}$calcd for $\mathrm{C}_{13} \mathrm{H}_{20} \mathrm{O}_{3} \mathrm{Na}$ :247.1310, found: 247.1301. A solution of the above hydroxy compound (300 $\mathrm{mg}, 1.34 \mathrm{mmol})$ in dry DCM $(5 \mathrm{~mL})$ was oxidized with oxalyl chloride $(0.29 \mathrm{~mL}, 3.35 \mathrm{mmol})$, DMSO $(0.32 \mathrm{~mL}, 4.69 \mathrm{mmol})$ and triethylamine $(0.56 \mathrm{~mL}, 4.02 \mathrm{mmol})$ according to the procedure described for preparation of the aldehyde 12, to afford the aldehyde 18 (247 $\mathrm{mg}, 83 \%)$ as a light yellow oil; IR: $v_{\max } 1720 \mathrm{~cm}^{-1} ;{ }^{1} \mathrm{H}$ NMR $\delta 1.4(1 \mathrm{H}, \mathrm{d}, J=7.8 \mathrm{~Hz}), 1.69(1 \mathrm{H}, \mathrm{d}, J=8.7 \mathrm{~Hz}), 1.80(1 \mathrm{H}$, dd, $J=12.3,3.3 \mathrm{~Hz}), 1.93(1 \mathrm{H}, \mathrm{d}, J=12 \mathrm{~Hz}), 2.93(1 \mathrm{H}, \mathrm{br} \mathrm{s}), 3.05(1 \mathrm{H}, \mathrm{br} \mathrm{s}), 3.37(3 \mathrm{H}$, s), $4.22(1 \mathrm{H}, \mathrm{d}, J=8.7 \mathrm{~Hz}), 4.47(1 \mathrm{H}, \mathrm{d}, J=6.9 \mathrm{~Hz}), 4.67(1 \mathrm{H}, \mathrm{d}, J=7.4 \mathrm{~Hz}), 5.25-5.40$ $(2 \mathrm{H}, \mathrm{m}), 5.83-5.95(1 \mathrm{H}, \mathrm{m}), 6.02(1 \mathrm{H}, \mathrm{m}), 6.20(1 \mathrm{H}, \mathrm{m}) ;{ }^{13} \mathrm{C}$ NMR $\delta 34.5\left(\mathrm{CH}_{2}\right), 43.1$ $(\mathrm{CH}), 47.1(\mathrm{CH}), 47.8\left(\mathrm{CH}_{2}\right), 56.3\left(\mathrm{CH}_{3}\right), 62.9(\mathrm{C}), 84.3(\mathrm{CH}), 93.9\left(\mathrm{CH}_{2}\right), 120.7\left(\mathrm{CH}_{2}\right)$, $134.6(\mathrm{CH}), 134.8(\mathrm{CH}), 139.9(\mathrm{CH}), 205.3(\mathrm{CHO})$; HRMS (ESI) m/z $(\mathrm{M}+\mathrm{Na})^{+}$calcd for $\mathrm{C}_{13} \mathrm{H}_{18} \mathrm{O}_{3} \mathrm{Na}$ :245.1154, found: 245.1149 .

\section{1-(2-(1-(Methoxymethoxy)allyl)bicyclo[2.2.1]hept-5-en-2-yl)pent-4-en-1-one}

19.

Following the procedure described for the preparation of the compound 13, the aldehyde $18(247 \mathrm{mg}, 1.13 \mathrm{mmol})$ in dry THF $(5 \mathrm{~mL})$ was treated with butenyl magnesium bromide [prepared from 4-bromo butene $(0.23 \mathrm{~mL}, 2.3 \mathrm{mmol})$ in THF $(10 \mathrm{~mL})$ and magnesium turnings (54 $\mathrm{mg}, 2.25 \mathrm{mmol}$ )] to afford the hydroxy compound as a 1:1 mixture. Column chromatography of the mixture gave one isomer of the alcohol $(101 \mathrm{mg}$, 
33\%); IR: $v_{\max } 3446 \mathrm{~cm}^{-1} ;{ }^{1} \mathrm{H}$ NMR $\delta 1.36(1 \mathrm{H}, \mathrm{d}, \mathrm{J}=8.7 \mathrm{~Hz}), 1.59-1.76(4 \mathrm{H}, \mathrm{m}), 1.81$ $(1 \mathrm{H}, \mathrm{dd}, J=12.3,3.8 \mathrm{~Hz}), 1.99-2.08(2 \mathrm{H}, \mathrm{m}), 2.35-2.37(1 \mathrm{H}, \mathrm{m}), 2.65(1 \mathrm{H}, \mathrm{br} \mathrm{s}), 2.91$ $(1 \mathrm{H}, \mathrm{br} \mathrm{s}), 3.08(1 \mathrm{H}, \mathrm{t}, J=10.1 \mathrm{~Hz}), 3.41(3 \mathrm{H}, \mathrm{s}), 4.31(1 \mathrm{H}, \mathrm{d}, J=9.1 \mathrm{~Hz}), 4.52(1 \mathrm{H}, \mathrm{d}, J$ $=7.0 \mathrm{~Hz}), 4.67(1 \mathrm{H}, \mathrm{d}, J=6.9 \mathrm{~Hz}), 4.95-5.07(2 \mathrm{H}, \mathrm{m}), 5.14-5.33(2 \mathrm{H}, \mathrm{m}), 5.94-5.96(1 \mathrm{H}$, m), $5.97(1 \mathrm{H}, \mathrm{m}), 6.01-6.06(1 \mathrm{H}, \mathrm{m}), 6.24(1 \mathrm{H}, \mathrm{dd}, J=5.48,3 \mathrm{~Hz}) .{ }^{13} \mathrm{C}$ NMR $\delta 31.9$ $\left(\mathrm{CH}_{2}\right), 35.3\left(\mathrm{CH}_{2}\right), 37.8\left(\mathrm{CH}_{2}\right), 42.7(\mathrm{CH}), 46.8\left(\mathrm{CH}_{2}\right), 48.4(\mathrm{CH}), 52.8(\mathrm{C}), 56.1\left(\mathrm{CH}_{3}\right)$, $80.2(\mathrm{CH}), 90.1(\mathrm{CH}), 93.7\left(\mathrm{CH}_{2}\right), 114.5\left(\mathrm{CH}_{2}\right), 119.6\left(\mathrm{CH}_{2}\right), 134.3(\mathrm{CH}), 135.6(\mathrm{CH})$, $138.9(\mathrm{CH}), 139.0(\mathrm{CH})$; and the other isomer $(100 \mathrm{mg}, 33 \%) ;{ }^{1} \mathrm{H}$ NMR $\delta 1.35(1 \mathrm{H}, \mathrm{d}, J=$ $9.3 \mathrm{~Hz}), 1.51(1 \mathrm{H}, \mathrm{dd}, J=3.9,8.2 \mathrm{~Hz}), 1.54-1.74(3 \mathrm{H}, \mathrm{m}), 2.0-2.1(2 \mathrm{H}, \mathrm{m}), 2.31-2.34$ $(1 \mathrm{H}, \mathrm{m}), 2.77(1 \mathrm{H}, \mathrm{br} \mathrm{s}), 3.11(1 \mathrm{H} \mathrm{br} \mathrm{s}), 3.18(1 \mathrm{H}, \mathrm{t}, J=9.5 \mathrm{~Hz}), 3.42(3 \mathrm{H}, \mathrm{s}), 4.34(1 \mathrm{H}$, d, $J=8.6 \mathrm{~Hz}), 4.54(1 \mathrm{H}, \mathrm{d}, J=6.9 \mathrm{~Hz}), 4.74(1 \mathrm{H}, \mathrm{d}, J=6.9 \mathrm{~Hz}), 4.91-5.04(2 \mathrm{H}, \mathrm{m})$, 5.15-5.31 (2H, m), 5.81-5.93 (2H, m), $6.14(1 \mathrm{H}, \mathrm{dd}, J=5.6,2.9 \mathrm{~Hz}), 6.31(1 \mathrm{H}, \mathrm{dd}, J=$ 5.5, $3 \mathrm{~Hz}) ;{ }^{13} \mathrm{C}$ NMR $\delta 31.9\left(\mathrm{CH}_{2}\right), 32.9\left(\mathrm{CH}_{2}\right), 33.6\left(\mathrm{CH}_{2}\right), 42.9(\mathrm{CH}), 46.8(\mathrm{CH}), 48.2$ $\left(\mathrm{CH}_{2}\right), 53.9(\mathrm{C}), 57.1\left(\mathrm{CH}_{3}\right), 79.6(\mathrm{CH}), 85.0(\mathrm{CH}), 94.7\left(\mathrm{CH}_{2}\right), 114.7\left(\mathrm{CH}_{2}\right), 118.97$ $\left(\mathrm{CH}_{2}\right), 137.5(\mathrm{CH}), 137.6(\mathrm{CH}), 137.9(\mathrm{CH}), 139.5(\mathrm{CH})$; HRMS (ESI) $\mathrm{m} / \mathrm{z}(\mathrm{M}+\mathrm{Na})^{+}$ calcd for $\mathrm{C}_{17} \mathrm{H}_{26} \mathrm{O}_{3} \mathrm{Na}: 301.1780$, found: 301.1773. The mixture of the hydroxy compounds obtained as above $(300 \mathrm{mg}, 1.08 \mathrm{mmol})$ in acetone $(3 \mathrm{~mL})$ was treated with Jones reagent ${ }^{1}(0.8 \mathrm{~mL})$ to afford the ketone $19(0.774 \mathrm{~g}, 78 \%)$; IR: $v_{\max } 1699 \mathrm{~cm}^{-1} ;{ }^{1} \mathrm{H}$ NMR $\delta 1.36(1 \mathrm{H}, \mathrm{dd}, J=8.7,1.8 \mathrm{~Hz}), 1.71(1 \mathrm{H}, \mathrm{d}, J=8.7 \mathrm{~Hz}), 1.93(2 \mathrm{H}, \mathrm{d}, J=9 \mathrm{~Hz})$, 2.20-2.38 (3H, m), 2.53-2.59 (1H, m), $2.89(1 \mathrm{H}, \mathrm{m}), 3.0(1 \mathrm{H}, \mathrm{m}), 3.40(3 \mathrm{H}, \mathrm{s}), 4.11(1 \mathrm{H}$, d, $J=8.7 \mathrm{~Hz}), 4.47(1 \mathrm{H}, \mathrm{d}, J=6.9 \mathrm{~Hz}), 4.66(1 \mathrm{H}, \mathrm{d}, J=7.7 \mathrm{~Hz}), 4.92-5.04(1 \mathrm{H}, \mathrm{m})$, 5.14-5.32 (2H, m), 5.68- $5.77(1 \mathrm{H}, \mathrm{m}), 5.95(1 \mathrm{H}, \mathrm{dd}, J=5.6,2.9 \mathrm{~Hz}), 6.17(1 \mathrm{H}, \mathrm{dd}, J=$ 5.6, $2.9 \mathrm{~Hz}) ;{ }^{13} \mathrm{C}$ NMR $\delta 27.8\left(\mathrm{CH}_{2}\right), 34.4\left(\mathrm{CH}_{2}\right), 42.3(\mathrm{CH}), 42.5\left(\mathrm{CH}_{2}\right), 46.7\left(\mathrm{CH}_{2}\right), 55.9$ $\left(\mathrm{CH}_{3}\right), 64.2(\mathrm{C}), 84.5(\mathrm{CH}), 93.5\left(\mathrm{CH}_{2}\right), 114.7\left(\mathrm{CH}_{2}\right), 119.6\left(\mathrm{CH}_{2}\right), 135.8(\mathrm{CH}), 137.9$ $(\mathrm{CH}), 138.9(\mathrm{CH}), 212.1(\mathrm{CO})$; HRMS (ESI) $\mathrm{m} / \mathrm{z}(\mathrm{M}+\mathrm{Na})^{+}$calcd for $\mathrm{C}_{17} \mathrm{H}_{24} \mathrm{O}_{3} \mathrm{Na}$ :299.1623, found: 299.1628 .

\section{2-Allyl-1-(2-(1-(methoxymethoxy)allyl)bicyclo[2.2.1]hept-5-en-2-yl)pent-4-en-1-one}

20. A solution of the ketone 21 (140 mg, $0.51 \mathrm{mmol})$ in THF $(1.5 \mathrm{~mL})$ was added drop wise to a magnetically stirred solution of LDA [prepared from diisopropylamine $(0.14$ $\mathrm{mL}, 1.02 \mathrm{mmol})$ in anhydrous THF $(1 \mathrm{~mL})$ and $\mathrm{n}-\mathrm{BuLi}(0.73 \mathrm{~mL}, 1.02 \mathrm{mmol}), 1.4 \mathrm{M}$ in hexane] at $-78^{\circ} \mathrm{C}$. The reaction mixture was then slowly raised to $-30^{\circ} \mathrm{C}$ and stirred at that 
temperature for $1 \mathrm{~h}$. The reaction mixture was then again cooled to $-78^{\circ} \mathrm{C}$ and to it was added HMPA $(0.1 \mathrm{~mL})$ followed by addition of a solution of allyl bromide $(0.05 \mathrm{~mL}$, $0.51 \mathrm{mmol})$. The reaction mixture was allowed to attain $-30^{\circ} \mathrm{C}$ and stirred for $2 \mathrm{~h}$ followed by quenching with $\mathrm{NH}_{4} \mathrm{Cl}(0.5 \mathrm{~mL})$. Usual work up of the reaction mixture followed by column chromatography (ether-petroleum ether 1:49) afforded the compound 20 (69 mg, $60 \%$ ) as an yellow oil; IR: $v_{\max } 1698 \mathrm{~cm}^{-1},{ }^{1} \mathrm{H}$ NMR $\delta 1.36-1.37(2 \mathrm{H}, \mathrm{m}) ; 1.56(2 \mathrm{H}$, $\mathrm{dd}, \mathrm{J}=12.6,3.4 \mathrm{~Hz}) ; 1.78-1.87(2 \mathrm{H}, \mathrm{m}), 2.13-2.34$ (3H, m); $2.74-2.80(2 \mathrm{H}, \mathrm{m}), 3.39$ $(3 \mathrm{H}, \mathrm{s}), 4.13(1 \mathrm{H}, \mathrm{d}, \mathrm{J}=8.4 \mathrm{~Hz}), 4.51(1 \mathrm{H}, \mathrm{d}, \mathrm{J}=6.7 \mathrm{~Hz}), 4.67(1 \mathrm{H}, \mathrm{d}, \mathrm{J}=7.0 \mathrm{~Hz}), 4.91-$ $5.05(3 \mathrm{H}, \mathrm{m}), 6.03-6.06(1 \mathrm{H}, \mathrm{m}), 6.15-6.17(1 \mathrm{H}, \mathrm{m}) ;{ }^{13} \mathrm{C} \mathrm{NMR} \delta 34.2\left(\mathrm{CH}_{2}\right), 34.8$ $\left(\mathrm{CH}_{2}\right), 35.3\left(\mathrm{CH}_{2}\right), 42.7(\mathrm{CH}), 47.6\left(\mathrm{CH}_{2}\right), 48.1(\mathrm{CH}), 48.4(\mathrm{CH}), 56.4\left(\mathrm{CH}_{3}\right), 65.8(\mathrm{C})$, $83.9(\mathrm{CH}), 94.1\left(\mathrm{CH}_{2}\right), 116.9\left(\mathrm{CH}_{2}\right), 116.93\left(\mathrm{CH}_{2}\right), 120\left(\mathrm{CH}_{2}\right), 134.9(\mathrm{CH}), 135.9(\mathrm{CH})$, $136.5(\mathrm{CH}), 139.5(\mathrm{CH}), 140.8(\mathrm{CH}), 215.3(\mathrm{CO})$; HRMS (ESI) m/z $(\mathrm{M}+\mathrm{Na})^{+}$calcd for $\mathrm{C}_{20} \mathrm{H}_{28} \mathrm{O}_{3} \mathrm{Na}$ :339.1936, found: 339.1944.

Synthesis of the bridged tricyclic ketone 21. Following the procedure described for metathesis of the compound 5, the compound 20 (70mg, $0.222 \mathrm{mmol})$ in DCM (15 mL) with the catalyst 7 (9 mg, $0.011 \mathrm{mmol})$ afforded the tricyclic compound 21 (45 $\mathrm{mg}, 70 \%)$ : IR: $v_{\max } 1697 \mathrm{~cm}^{-1} ;{ }^{1} \mathrm{H}$ NMR $\delta 1.40(1 \mathrm{H}, \mathrm{dd}, J=12.6,3.6 \mathrm{~Hz}), 1.50(1 \mathrm{H}, \mathrm{d}, J=8.7 \mathrm{~Hz})$, $1.70(1 \mathrm{H}, \mathrm{d}, J=8.7 \mathrm{~Hz}), 1.93(1 \mathrm{H}, \mathrm{dd}, J=12.6,2.7 \mathrm{~Hz}), 2.33-2.37(2 \mathrm{H}, \mathrm{m}), 2.41-2.48$ $(2 \mathrm{H}, \mathrm{m}), 2.54-2.57(1 \mathrm{H}, \mathrm{m}), 2.81(1 \mathrm{H}, \mathrm{br} \mathrm{s}), 3.42(3 \mathrm{H}, \mathrm{s}), 3.71(1 \mathrm{H}, \mathrm{m}), 4.10(1 \mathrm{H}, \mathrm{d}, J=$ $8.1 \mathrm{~Hz}), 4.52(1 \mathrm{H}, \mathrm{d}, J=6.6 \mathrm{~Hz}), 4.69(1 \mathrm{H}, \mathrm{d}, J=6.6 \mathrm{~Hz}), 5.19-5.28(2 \mathrm{H}, \mathrm{m}), 5.42-$ $5.48(1 \mathrm{H}, \mathrm{m}), 5.58-5.63(2 \mathrm{H}, \mathrm{m}), 6.01(1 \mathrm{H}, \mathrm{dd}, J=5.7,2.7 \mathrm{~Hz}), 6.18(1 \mathrm{H}, \mathrm{dd}, J=5.7$, $\mathrm{Hz}) ;{ }^{13} \mathrm{C}$ NMR $\delta 34.6\left(\mathrm{CH}_{2}\right), 39.1\left(\mathrm{CH}_{2}\right), 39.7\left(\mathrm{CH}_{2}\right), 42.6(\mathrm{CH}), 47.0(\mathrm{CH}), 47.16\left(\mathrm{CH}_{2}\right)$, $47.22(\mathrm{CH}), 56.1(\mathrm{CH}), 65.7(\mathrm{C}), 83.3\left(\mathrm{CH}_{3}\right), 93.8\left(\mathrm{CH}_{2}\right), 119.8\left(\mathrm{CH}_{2}\right), 128.9(\mathrm{CH}), 129.2$ $(\mathrm{CH}), 134.1(\mathrm{CH}), 134.6(\mathrm{CH}), 140.2(\mathrm{CH}), 217.9(\mathrm{CO}) ; \mathrm{HRMS}(\mathrm{ESI}) \mathrm{m} / \mathrm{z}(\mathrm{M}+\mathrm{Na})^{+}$ calcd for $\mathrm{C}_{18} \mathrm{H}_{24} \mathrm{O}_{3} \mathrm{Na}: 311.1623$, found: 311.1628 .

1-(2Allylbicyclo[2.2.1]hept-5-en-2-yl)but-3-en-1-one 26. A solution of allyl bromide (3.49 mL, $32.92 \mathrm{mmol})$ in THF $(20 \mathrm{~mL})$ was added drop wise to a stirred suspension of commercial zinc dust $(1.44 \mathrm{~g}, 21.95 \mathrm{mmol})$ in THF $(10 \mathrm{~mL})$ at $\mathrm{rt}$, and the mixture was stirred for $1 \mathrm{~h}$ to produce a clear solution. A solution of the aldehyde 12 (3 g, $18.29 \mathrm{mmol})$ in THF $(20 \mathrm{~mL})$ was added drop wise to this solution at $\mathrm{rt}$ and stirred for $1 \mathrm{~h}$. The reaction mixture was then cooled to $0{ }^{\circ} \mathrm{C}$ and quenched by addition of a few drops of 
water. Usual work up of the reaction mixture followed by column chromatography using ether-petroleum ether (1:6) as eluent afforded the allyl addition product $(0.856 \mathrm{~g}, 68 \%)$ as a mixture of the two diastereomers; IR: $v_{\max } 3447 \mathrm{~cm}^{-1} ;{ }^{1} \mathrm{H}$ NMR (for the mixture) $\delta 1.27$ - $1.29(1 \mathrm{H}, \mathrm{m}), 1.33-1.35(1 \mathrm{H}, \mathrm{m}), 1.39-1.44(2 \mathrm{H}, \mathrm{m}), 2.18-2.38(2 \mathrm{H}, \mathrm{m}), 2.42-2.46$ $(2 \mathrm{H}, \mathrm{m}), 2.52(1 \mathrm{H}$, br s), 2.79-2.93 $(1 \mathrm{H}, \mathrm{m}), 3.23-3.27(1 \mathrm{H}, \mathrm{m}), 5.02-5.13(4 \mathrm{H}, \mathrm{m})$, 5.78-5.84 $(1 \mathrm{H}, \mathrm{m}), 5.98-6.01(1 \mathrm{H}, \mathrm{m}), 6.09-6.17(2 \mathrm{H}, \mathrm{m}) ;{ }^{13} \mathrm{C}$ NMR (for the mixture) $\delta$ $35.9\left(\mathrm{CH}_{2}\right), 36.9\left(\mathrm{CH}_{2}\right), 38.3\left(\mathrm{CH}_{2}\right), 38.4\left(\mathrm{CH}_{2}\right), 40.5\left(\mathrm{CH}_{2}\right), 40.54\left(\mathrm{CH}_{2}\right), 42.4(\mathrm{CH})$, $42.9(\mathrm{CH}), 46.8\left(\mathrm{CH}_{2}\right), 47.8\left(\mathrm{CH}_{2}\right), 47.8(\mathrm{CH}), 49.7(\mathrm{CH}), 50.3(\mathrm{C}), 50.4(\mathrm{C}) ; 78.6(\mathrm{CH})$, $79.1(\mathrm{CH}), 116.1\left(\mathrm{CH}_{2}\right), 116.2\left(\mathrm{CH}_{2}\right), 116.87\left(\mathrm{CH}_{2}\right), 116.9\left(\mathrm{CH}_{2}\right), 134.2(\mathrm{CH}), 135.4$ $(\mathrm{CH}), 136.4(\mathrm{CH}), 136.5(\mathrm{CH}), 136.9(\mathrm{CH}), 137.7(\mathrm{CH}), 137.7(\mathrm{CH}), 138.3(\mathrm{CH}) ;$ HRMS (ESI) $\mathrm{m} / \mathrm{z}(\mathrm{M}+\mathrm{Na})^{+}$calcd for $\mathrm{C}_{14} \mathrm{H}_{20} \mathrm{ONa}: 227.1412$, found:227.1418. The hydroxy compound thus obtained $(1.5 \mathrm{~g}, 7.35 \mathrm{mmol})$ in acetone $(5.6 \mathrm{~mL})$, was oxidized with Jones reagent $^{1}(2 \mathrm{ml})$ to afford the ketone $26(0.832 \mathrm{~g}, 84 \%)$ as a colorless liquid. IR: $v_{\max } 1706$ $\mathrm{cm}^{-1} ;{ }^{1} \mathrm{H}$ NMR $\delta$ 1.44-1.51 (2H, m), $1.63(1 \mathrm{H}, \mathrm{d}, J=8.7 \mathrm{~Hz}), 1.96(1 \mathrm{H}, \mathrm{dd}, J=12.1,2.6$ $\mathrm{Hz}), 2.40$ (2H, dd, $J=14.3,6.9 \mathrm{~Hz}), 2.65(2 \mathrm{H}, \mathrm{dd}, J=14.3,7.5 \mathrm{~Hz}), 2.85-2.89(1 \mathrm{H}, \mathrm{m})$, 3.16 - $3.20(1 \mathrm{H}, \mathrm{m}), 4.99-5.16(4 \mathrm{H}, \mathrm{m}), 5.61(1 \mathrm{H}, \mathrm{m}), 5.84-5.89(1 \mathrm{H}, \mathrm{m}), 5.91-5.95(1 \mathrm{H}$, m), $6.13(1 \mathrm{H}, \mathrm{dd}, J=5.6,3 \mathrm{~Hz}) ;{ }^{13} \mathrm{C}$ NMR $\delta 33.8\left(\mathrm{CH}_{2}\right), 42.7(\mathrm{CH}), 43.4\left(\mathrm{CH}_{2}\right), 44.2$ $\left(\mathrm{CH}_{2}\right), 47.2\left(\mathrm{CH}_{2}\right), 50.2(\mathrm{CH}), 61.1(\mathrm{C}), 117.2\left(\mathrm{CH}_{2}\right), 117.6\left(\mathrm{CH}_{2}\right), 131.5(\mathrm{CH}), 133.7$ $(\mathrm{CH}), 134.01(\mathrm{CH}), 138.6(\mathrm{CH}), 210.1(\mathrm{CO})$; HRMS $(\mathrm{ESI}) \mathrm{m} / \mathrm{z}(\mathrm{M}+\mathrm{Na})^{+}$calcd for $\mathrm{C}_{14} \mathrm{H}_{18} \mathrm{ONa}: 225.1255$, found: 225.1259 .

1-(2-Allylbicyclo[2.2.1]hept-5-en-2-yl)-2-vinylpent-4-en-1-one 27. Following the procedure used for allylation of the compound 13, the ketone 26 (500 mg, $2.48 \mathrm{mmol}$ ) was treated with LDA [prepared from diisopropyl amine $(0.52 \mathrm{~mL}, 3.71 \mathrm{mmol})$ in anhydrous THF $(5 \mathrm{~mL})$ and $\mathrm{nBuLi}(2.3 \mathrm{~mL}, 3.71 \mathrm{mmol}) 1.6 \mathrm{M}$ in hexane] and HMPA $(0.2 \mathrm{~mL})$ to afford the ketone $27(0.779 \mathrm{~g}, 65 \%)$ as colorless oil. IR: $v_{\max } 1706 \mathrm{~cm}^{-1} ;{ }^{1} \mathrm{H}$ NMR $\delta 1.42-1.52(2 \mathrm{H}, \mathrm{m}), 1.61(1 \mathrm{H}, \mathrm{d}, J=8.7 \mathrm{~Hz}), 1.87(1 \mathrm{H}, \mathrm{dd}, J=12.1,2.4 \mathrm{~Hz}), 2.15-$ $2.29(2 \mathrm{H}, \mathrm{m}), 2.49(1 \mathrm{H}, \mathrm{dd}, J=14.9,6.8 \mathrm{~Hz}), 2.64(1 \mathrm{H}, \mathrm{dd}, J=14.8,7.2 \mathrm{~Hz}), 2.83(1 \mathrm{H}$, br s), $2.9(1 \mathrm{H}$, br s), $3.38(1 \mathrm{H}, \mathrm{m}), 4.97-5.13(6 \mathrm{H}, \mathrm{m}), 5.55-5.71(3 \mathrm{H}, \mathrm{m}), 5.97-5.99$ $(1 \mathrm{H}, \mathrm{m}), 6.05-6.08(1 \mathrm{H}, \mathrm{m}) ;{ }^{13} \mathrm{C}$ NMR $\delta 34.1\left(\mathrm{CH}_{2}\right), 36.5\left(\mathrm{CH}_{2}\right), 42.3(\mathrm{CH}), 43.0\left(\mathrm{CH}_{2}\right)$, $46.8\left(\mathrm{CH}_{2}\right), 49.1\left(\mathrm{CH}_{2}\right), 54.0(\mathrm{CH}), 61.7(\mathrm{C}), 116.6\left(\mathrm{CH}_{2}\right), 117.0\left(\mathrm{CH}_{2}\right), 117.4\left(\mathrm{CH}_{2}\right)$, 
$134.2(\mathrm{CH}), 135.5(\mathrm{CH}), 135.8(\mathrm{CH}), 137.3(\mathrm{CH}), 137.5(\mathrm{CH}), 212.5(\mathrm{CO})$; HRMS (ESI) $\mathrm{m} / \mathrm{z}(\mathrm{M}+\mathrm{Na})^{+}$calcd for $\mathrm{C}_{17} \mathrm{H}_{22} \mathrm{ONa}: 265.1568$, found: 265.1566 .

Synthesis of the bridged tricyclic ketone 28. Following the procedure described for metathesis of compound 5, the compound 27 ( $80 \mathrm{mg}, 0.33 \mathrm{mmol}$ ) in DCM $(15 \mathrm{~mL})$ with the catalyst 7 ( $10 \mathrm{mg}, 0.012 \mathrm{mmol}$ ) afforded the tricyclic compound 28 (28 $\mathrm{mg}, 40 \%)$ : IR: $v_{\max }$ 1701. $\mathrm{cm}^{-1} ;{ }^{1} \mathrm{H}$ NMR $\delta 1.44-1.48(1 \mathrm{H}, \mathrm{m}), 1.59-1.67(3 \mathrm{H}, \mathrm{m}), 2.14(2 \mathrm{H}, \mathrm{dd}, J=2.6$, $11.9 \mathrm{~Hz}), 2.24-2.35(2 \mathrm{H}, \mathrm{m}), 2.56-2.60(1 \mathrm{H}, \mathrm{m}), 2.86-2.98(1 \mathrm{H}, \mathrm{m}), 3.66(1 \mathrm{H}, \mathrm{m}), 5.05$ $(2 \mathrm{H}, \mathrm{dd}, J=13.6,2.2 \mathrm{~Hz}), 5.80-5.88(3 \mathrm{H}, \mathrm{m}), 5.97-6.06(1 \mathrm{H}, \mathrm{m}), 6.16(1 \mathrm{H}, \mathrm{dd}, J=$ 5.64, $3 \mathrm{~Hz}) ;{ }^{13} \mathrm{C}$ NMR $\delta 33.7\left(\mathrm{CH}_{2}\right), 36.4\left(\mathrm{CH}_{2}\right), 37.03\left(\mathrm{CH}_{2}\right), 42.8(\mathrm{CH}), 46.8\left(\mathrm{CH}_{2}\right)$, $49.7(\mathrm{CH}), 52.4(\mathrm{CH}), 62.1(\mathrm{C}), 115.05\left(\mathrm{CH}_{2}\right), 128.2(\mathrm{CH}), 129.9(\mathrm{CH}), 132.9(\mathrm{CH})$, $137.3(\mathrm{CH}), 139.13(\mathrm{CH}), 213.1(\mathrm{CO})$; HRMS (ESI) $\mathrm{m} / \mathrm{z}(\mathrm{M}+\mathrm{Na})^{+}$calcd for $\mathrm{C}_{15} \mathrm{H}_{18} \mathrm{ONa}$ :237.1255, found: 237.1250 .

1-(Bicyclo[2.2.1]hept-5-en-2-yl)prop-2-en-1-one 32. The aldehyde 31 (3 g, 24.6 mmol) in dry THF $(15 \mathrm{~mL})$ was treated with vinyl magnesium bromide [prepared from vinyl bromide (3.3 g, $31 \mathrm{mmol})$ in THF $(40 \mathrm{~mL})$ and magnesium turnings $(1.8 \mathrm{~g}, 73.77 \mathrm{mmol})$ ] to afford the vinyl addition product $(3.2 \mathrm{~g}, 85 \%)$ as a $1: 1$ diasteriomeric mixture; IR: $v_{\max }$ $3450 \mathrm{~cm}^{-1} ;{ }^{1} \mathrm{H}$ NMR (for the mixture) $\delta 1.19-1.22(1 \mathrm{H}, \mathrm{m}), 1.35-1.43(1 \mathrm{H}, \mathrm{m}), 1.65-$ $1.72(1 \mathrm{H}, \mathrm{m}), 1.85-1.86(1 \mathrm{H}, \mathrm{m}) ; 2.01-2.12(1 \mathrm{H}, \mathrm{m}), 2.22(\mathrm{OH}$ br s), 2.71-2.80 $(1 \mathrm{H}, \mathrm{m})$, $3.02(1 \mathrm{H}$, br s), 3.36-3.45 $(1 \mathrm{H}, \mathrm{m}), 4.99-5.17(2 \mathrm{H}, \mathrm{m}), 5.75-5.88(1 \mathrm{H}, \mathrm{m}), 5.99-6.02$ $(1 \mathrm{H}, \mathrm{m}), 6.12-6.13(1 \mathrm{H}, \mathrm{m}) ;{ }^{13} \mathrm{C}$ NMR (for the mixture) $\delta 29.2\left(\mathrm{CH}_{2}\right), 29.8\left(\mathrm{CH}_{2}\right), 42.2$ $(\mathrm{CH}), 42.7(\mathrm{CH}), 43.9(\mathrm{CH}), 44.6(\mathrm{CH}), 45.3(\mathrm{CH}), 46.3(\mathrm{CH}), 49.3\left(\mathrm{CH}_{2}\right), 49.6\left(\mathrm{CH}_{2}\right)$, $76.9(\mathrm{CH}), 78.0(\mathrm{CH}), 114.7\left(\mathrm{CH}_{2}\right), 114.9\left(\mathrm{CH}_{2}\right), 132.1(\mathrm{CH}), 132.6(\mathrm{CH}), 137.7(\mathrm{CH})$, $137.8(\mathrm{CH}), 140.7(\mathrm{CH}), 140.8(\mathrm{CH})$; HRMS (ESI) $\mathrm{m} / \mathrm{z}(\mathrm{M}+\mathrm{Na})^{+}$calcd for $\mathrm{C}_{10} \mathrm{H}_{14} \mathrm{ONa}$ :173.0942, found: 173.0935 . The above alcohol $(500 \mathrm{mg}, 3.33 \mathrm{mmol})$ in acetone $(5 \mathrm{~mL})$ was treated with Jones reagent ${ }^{1}(1.2 \mathrm{~mL})$ to afford the ketone $32(346 \mathrm{mg}, 70 \%)$ : IR: $v_{\max }$ $1697 \mathrm{~cm}^{-1} ;{ }^{1} \mathrm{H}$ NMR $\delta 1.29(1 \mathrm{H}, \mathrm{d}, J=8.1 \mathrm{~Hz}), 1.39(1 \mathrm{H}, \mathrm{dd}, J=1.4,7.8 \mathrm{~Hz}), 1.44-1.50$ $(1 \mathrm{H}, \mathrm{m}), 1.71-1.78(1 \mathrm{H}, \mathrm{m}), 2.85(1 \mathrm{H}, \mathrm{br} \mathrm{s}), 3.16-3.21(2 \mathrm{H}, \mathrm{m}), 5.65(1 \mathrm{H}, \mathrm{dd}, J=10.5$, $1.1 \mathrm{~Hz}), 5.74(1 \mathrm{H}, \mathrm{dd}, J=5.4,2.5 \mathrm{~Hz}), 6.07(1 \mathrm{H}, \mathrm{dd}, J=5.5,3.0 \mathrm{~Hz}), 6.16(1 \mathrm{H}, \mathrm{dd}, J=$ $17.5,1.10 \mathrm{~Hz}), 6.40(1 \mathrm{H}, \mathrm{dd}, J=17.5,10.5 \mathrm{~Hz}) ;{ }^{13} \mathrm{C} \mathrm{NMR} \delta 28.3\left(\mathrm{CH}_{2}\right), 43.2(\mathrm{CH}), 46.5$ $(\mathrm{CH}), 50.1(\mathrm{CH}), 50.2\left(\mathrm{CH}_{2}\right), 127.7\left(\mathrm{CH}_{2}\right), 131.9(\mathrm{CH}), 136.1(\mathrm{CH}), 137.8(\mathrm{CH}), 200.9$ (CO); HRMS (ESI) $\mathrm{m} / \mathrm{z}(\mathrm{M}+\mathrm{H})^{+}$calcd for $\mathrm{C}_{10} \mathrm{H}_{13} \mathrm{O}: 149.0966$, found: 149.0975 . 
Diels-Alder reaction of the enone 32. To a solution of the enone 32 (200 $\mathrm{mg}, 1.35$ $\mathrm{mmol})$ in dry DCM $(10 \mathrm{~mL})$ freshly distilled cyclopentadiene $(445 \mathrm{mg}, 6.75 \mathrm{mmol})$ was added drop wise in ice-cold condition. Stirring was continued for $1 \mathrm{~h}$ in $0{ }^{\circ} \mathrm{C}$ and $13 \mathrm{~h}$ at rt. The solvent was removed under reduced pressure and the white residue was purified by column chromatography (ether - petroleum ether 1:19) to afford an inseparable mixture of the adducts 33 and $34(220 \mathrm{mg}, 76 \%)$ as a white solid, mp 112-118 ${ }^{\circ} \mathrm{C}$ IR: $v_{\max } 1695 \mathrm{~cm}^{-1} ;{ }^{1} \mathrm{H}$ NMR (for the mixture) $\delta$ 1.29-1.36 (2H, m), 1.37-1.46 (2H, m), 1.49$1.55(1 \mathrm{H}, \mathrm{m}), 1.65-1.85(2 \mathrm{H}, \mathrm{m}), 2.89$ (1H, br s), 3.03-3.14 (2H, m), $3.22-3.31(2 \mathrm{H}, \mathrm{m})$, 5.77- $5.90(2 \mathrm{H}, \mathrm{m}), 6.10-6.14(2 \mathrm{H}, \mathrm{m}) ;{ }^{13} \mathrm{C} \mathrm{NMR}$ ( for the mixture) $\delta 27.6\left(\mathrm{CH}_{2}\right), 28.9$ $\left(\mathrm{CH}_{2}\right), 42.7(\mathrm{CH}), 42.9(\mathrm{CH}), 45.8(\mathrm{CH}), 46.5(\mathrm{CH}), 49.9\left(\mathrm{CH}_{2}\right), 50.1\left(\mathrm{CH}_{2}\right), 50.3(\mathrm{CH})$, $51.1(\mathrm{CH}), 131.1(\mathrm{CH}), 132.3(\mathrm{CH}), 137.3(\mathrm{CH}), 137.9(\mathrm{CH}), 210.8(\mathrm{CO}), 212.2(\mathrm{CO})$; HRMS (ESI) m/z $(\mathrm{M}+\mathrm{Na})^{+}$calcd for $\mathrm{C}_{15} \mathrm{H}_{18} \mathrm{ONa}: 237.1255$, found: 237.1254.

\section{Metathesis of the mixture of the compounds 33 and 34 . Synthesis of the tricyclic}

compounds $35 \& 36$. Following the procedure described for metathesis of the compound 5, the inseparable mixture of the compounds 33 and 34 (83mg, $0.39 \mathrm{mmol})$ in DCM (15 $\mathrm{mL})$ with the catalyst $7(16 \mathrm{mg}, 0.019 \mathrm{mmol})$ afforded a mixture of the tricyclic compounds 35 and 36. Column chromatography of this mixture gave the pure ketone 35 (22 mg, 23\%); IR: $v_{\max } 1697.2 \mathrm{~cm}^{-1}$; ${ }^{1} \mathrm{H}$ NMR $\delta 1.48(2 \mathrm{H}, \mathrm{dd}, J=23.2,11.3 \mathrm{~Hz}), 1.89$ $(2 \mathrm{H}, \mathrm{dd}, J=22.2,10.5 \mathrm{~Hz}), 1.98-2.16(4 \mathrm{H}, \mathrm{m}), 2.50-2.62(2 \mathrm{H}, \mathrm{m}), 2.92-3.0(2 \mathrm{H}, \mathrm{m}), 3.32$ $(2 \mathrm{H}, \mathrm{dd}, J=8.5,18.4 \mathrm{~Hz}), 4.97-5.10$ (4H, m), 5.62 (2H, d, J=2.8 Hz), 5.84-5.95 (2H, m); ${ }^{13} \mathrm{C}$ NMR $\delta 35.1\left(\mathrm{CH}_{2}\right), 40.7\left(\mathrm{CH}_{2}\right), 41.1(\mathrm{CH}), 44.2(\mathrm{CH}), 54.1(\mathrm{CH}), 114.0\left(\mathrm{CH}_{2}\right), 132.4$ $(\mathrm{CH}), 141.8(\mathrm{CH}), 211.8(\mathrm{CO})$; HRMS (ESI) $\mathrm{m} / \mathrm{z}(\mathrm{M}+\mathrm{Na})^{+}$calcd for $\mathrm{C}_{17} \mathrm{H}_{22} \mathrm{ONa}$ : 265.1568, found: 265.1585 , and the pure ketone 36 (57mg, 45\%); IR: $v_{\max } 1703 \mathrm{~cm}^{-1} ;{ }^{1} \mathrm{H}$ NMR $\delta$ 1.25-1.32 (2H, m), 1.41-1.46 (2H, m), 1.78-1.87 (2H, m), 1.90-1.98 (2H, m), 2.47-2.58 (2H, m), 2.88-2.92 (1H, m), 2.95-3.05 (2H, m), 3.21-3.24 (1H, m), 3.32-3.40 $(1 \mathrm{H}, \mathrm{m}), 4.86-5.04(4 \mathrm{H}, \mathrm{m}), 5.51-5.63(1 \mathrm{H}, \mathrm{m}), 5.69(1 \mathrm{H}, \mathrm{dd}, J=5.4,2.6 \mathrm{~Hz}), 5.74-5.89$ $(1 \mathrm{H}, \mathrm{m}), 6.14(1 \mathrm{H}, \mathrm{dd}, J=5.4,3.0 \mathrm{~Hz}) ;{ }^{13} \mathrm{C} \mathrm{NMR} \delta 26.9\left(\mathrm{CH}_{2}\right), 34.9\left(\mathrm{CH}_{2}\right), 39.6\left(\mathrm{CH}_{2}\right)$, $43.0(\mathrm{CH}), 43.9(\mathrm{CH}), 46.3(\mathrm{CH}), 47.7(\mathrm{CH}), 50.2\left(\mathrm{CH}_{2}\right), 52.9(\mathrm{CH}), 53.5(\mathrm{CH}), 113.4$ $\left(\mathrm{CH}_{2}\right), 115.2\left(\mathrm{CH}_{2}\right), 130.9(\mathrm{CH}), 137.9(\mathrm{CH}), 139.6(\mathrm{CH}), 142.2(\mathrm{CH}), 212.5(\mathrm{CO})$; HRMS (ESI) m/z $(\mathrm{M}+\mathrm{Na})^{+}$calcd for $\mathrm{C}_{17} \mathrm{H}_{22} \mathrm{ONa}: 265.1568$, found: 265.1564 . 


\section{$\underline{\text { X-Ray crystal data for compound } 8}$}

See (fig 1) and Crystal data: Refined formula $\mathrm{C}_{18} \mathrm{H}_{22} \mathrm{O}_{3}$, Formula weight $\mathrm{M}_{\mathrm{r}}=286.36$, Crystal system Triclinic, Space group P-1 (No. 2), $\mathrm{a}=10.2279(13, \mathrm{~b}=10.6309(13)$, $\mathrm{c}=$ 15.3920(19) $\AA, \alpha=80.421(2)^{\circ}, \beta=71.857(2)^{\circ}, \gamma=89.308(2)^{\circ}$, Volume $(V)=1566.8(3) \AA^{3}$, No of the formula units in the unit cell $Z=2$, Calculated density $\rho_{\text {calcd }}=1.214 \mathrm{~g} \mathrm{~cm}^{-3}$, linear absorption coefficient $\mu(\mathrm{Mo}-\mathrm{K} \alpha)=\left(0.081 \mathrm{~mm}^{-1}\right)$, radiation and wavelength $\mathrm{MoK} \alpha(\lambda=$ $0.71073 \AA$ ) , temperature of measurement $\mathrm{T}=298 \mathrm{~K}, \mathrm{~F}(000)=616, \theta_{\min }=2.1^{\circ}, \theta_{\max }=28.3^{\circ}$, no. of measured reflection 9414, no. of independent reflection $6894, R_{\text {int }}=0.020, R_{1}=$ $0.0590, \mathrm{wR} 2=0.1872$, residual electron density $\max$ and $\min -0.15,0.28 \mathrm{e} \AA^{-3}$. The structure was solved by direct methods (SHELXS-97) and refined by full- matrix least squares using SHELXL-97 with anisotropic thermal parameters for non-hydrogen atoms. Hydrogen atoms were partly refined with isotropic thermal parameters, and partly placed in calculated position. 